\title{
STA-MCA Anastomosis and Collateral Circulation
}

\author{
Yoshikazu Iwata, Toru Hayakawa*, Keiji Shimizu, \\ Jiro Mukawa, Heitaro Mogami, Tadaatsu Nukada**, \\ Kazufumi Kimura**, Shotaro YonedA**, Takashi TAKANO** \\ and Masanori Miтомо*** \\ Department of Neurosurgery, \\ Osaka University Medical School, Osaka 553 \\ * Department of Neurosurgery, \\ The Center for Adult Diseases \\ ** Department of Internal Medicine, \\ Osaka University Medical School \\ ***Department of Radiology, \\ Osaka University Medical School
}

\begin{abstract}
Summary
Two representative cases of anastomosis of the superficial temporary artery (STA) to the middle cerebral artery (MCA) for the treatment of focal cerebral ischemia are presented and the changes in collateral circulation following the surgery are discussed based on twenty cases treated in this way.

Redistribution of cerebral circulation took place following the surgery. Although the collateral pathway constructed microsurgically was narrow and slender in the early postoperative period, it dilated with time in a majority of the cases. On the other hand, some of preexisting extra- to intracranial collateral channels atrophied and narrowed following the surgery.

The role of the microsurgically constructed bypass might be of limited value in the early postoperative period. Spontaneously developed collateral channels must, therefore, be preserved to operate safely for prevention of cerebral ischemic attacks particularly in this period.

Our findings indicate that sacrificing the anterior branch of the STA for STA-MCA anastomosis might be risky in certain cases in which the anterior branch was functioning as one of the main preexisting extra- to intracranial collateral routes. In such cases only the posterior branch of STA should be used as a donor artery.
\end{abstract}

Key words: Microvascular surgery, cerebral ischemia, STA-MCA anastomosis, collateral circulation

The microvascular surgical technique was introduced to neurosurgery by Jacobson, Donaghy and Yasargil ${ }^{3,16}$ in 1967 and anastomosis of the superficial temporal artery (STA) to the middle cerebral artery (MCA) was developed by Yasargi1 ${ }^{15)}$ and Donaghy as a new therapeutic approach for ischemic cerebral vascular diseases. Although this surgical procedure has been performed universally in recent years, there still remain some discrepancies in the criteria for surgical indication. ${ }^{9)}$

In an attempt to decide the indication of this surgery and to evaluate the surgical effect, a variety of clinical and laboratory examinations have been carried out both pre- and postoperatively in 20 patients operated on (22 operations) in our Department of Neurosurgery. These included neurological examinations, serial cerebral angiography, CT scanning, measurement of regional cerebral blood flow (rCBF) and its functional image, extracranial blood flow measurement by ultrasonic Doppler technique, ophthalmodynamometry, radioisotopic scintigraphy, electroencephalography, cerebrospinal fluid examinations, and psychometric tests. ${ }^{6)}$

The patients were followed up every 3 to 6 months up to 4 years after surgery.

The purpose of this paper is to present the 
changes of collateral circulation and hemodynamics after STA-MCA anastomosis in two representative cases in which long term followup was performed, and to discuss the operative techniques and the efficacy of this surgery for the treatment of focal cerebral ischemia, based upon the results of our entire series.

\section{Case Presentation}

\section{Case 1}

A 54 year-old left-handed male had been in good health until the end of 1975 when he experienced blurred vision of the right eye followed by sudden weakness of his left lower extremity and a speech disturbance lasting for a few minutes. Since then he had several similar episodes. Weakness of his extremities occurred usually on the left side, limited once to the arm and once to the leg. Episodes of black out of vision, which cleared within a few minutes, were noted on either side, and became more frequent. He was admitted to the Osaka University Hospital on March 17, 1976. Neurological examinations on admission were negative. Vascular bruits were audible in the right cervical and orbital areas on both sides. Funduscopy revealed venous stasis retinopathy of the left eye. Laboratory data showed positive sugar in urine and $126 \mathrm{mg} / \mathrm{dl}$ of fasting blood sugar. Doppler flowmetry showed increased left external carotid flow and retrograde blood flow of the left ophthalmic artery. Cerebral angiograms demonstrated complete occlusion of the left internal carotid artery at the cervical level and stenosis of the right internal carotid artery at the siphon level (Fig. 1). Extra- to intracranial collateral circulation was fairly well developed through the left ophthalmic artery with retrograde flow. No abnormality was found in CT scanning. Fig. 2 shows an anteroposterior view of the rCBF functional image $^{13)}$ following intracarotid injection of Xenon-133. By digital compression of the left STA, rCBF in the territory of the left MCA clearly decreased. These findings indicated that the blood flow through the STA might play a role as a collateral blood supply into the ischemic part of the brain and influence the whole cerebral circulation. Serial angiograms revealed that the main route of this collateral was from the anterior branch of the left STA to the left intracranial internal carotid artery via the left ophthalmic artery.

Left STA-MCA anastomosis ${ }^{5)}$ was performed on May 18, 1976. Based on the preoperative findings, the posterior branch of the left STA was selectively used as a donor artery and the
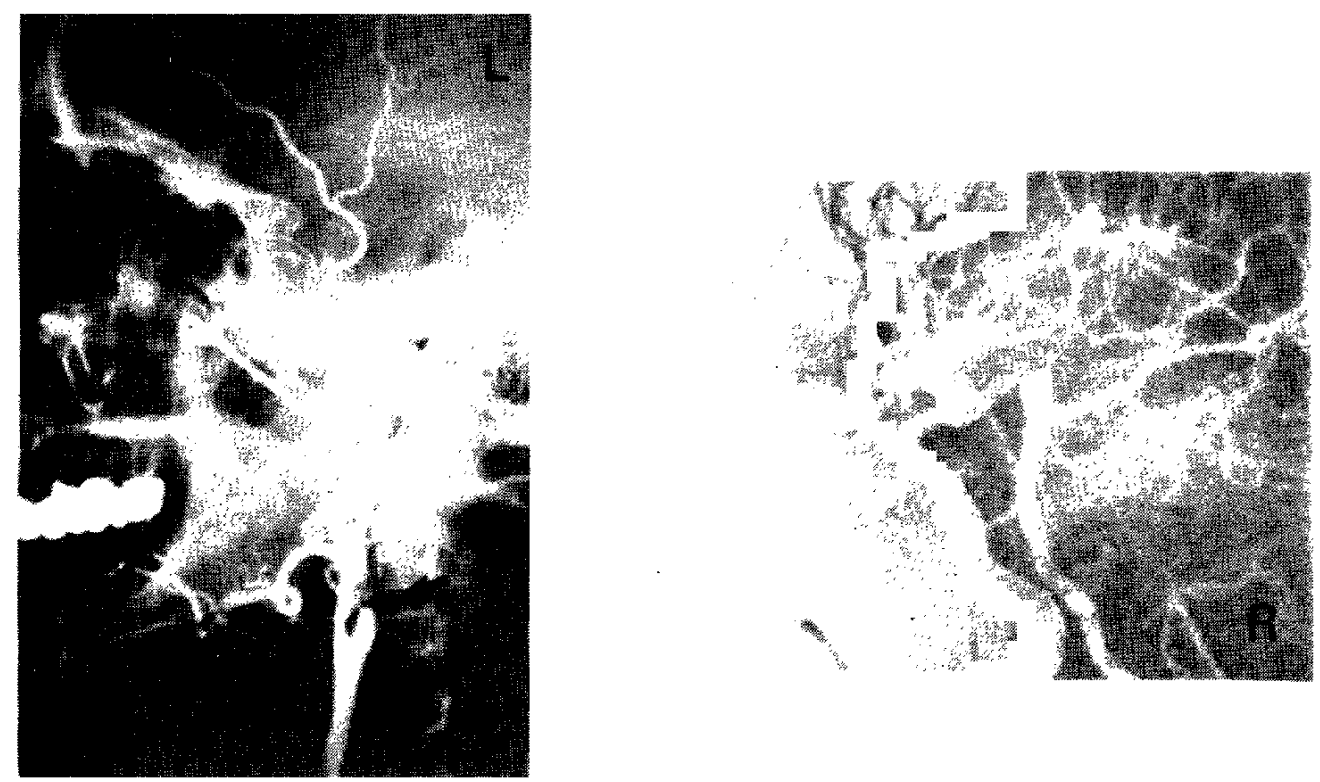

Fig. 1 Cerebral angiograms in Case 1 ( 54 year-old male) showing complete occlusion of the cervical internal carotid artery on the left side, and stenosis at the cavernous portion of the internal carotid artery on the right side. 
control

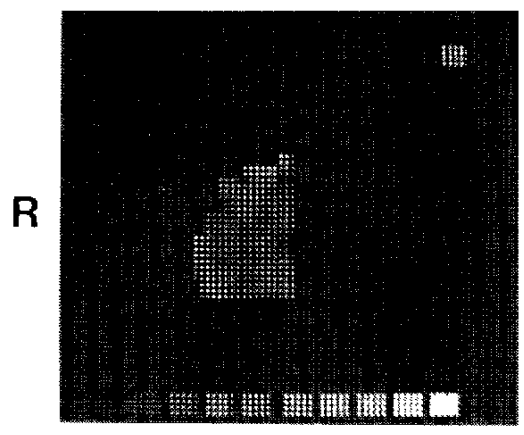

L-STA comp.

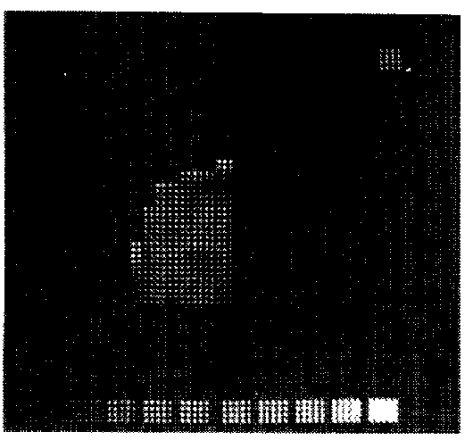

decreased area

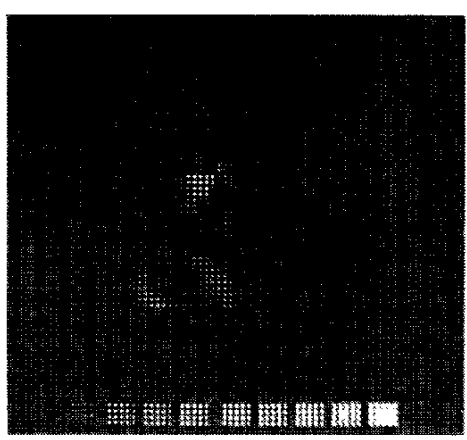

control

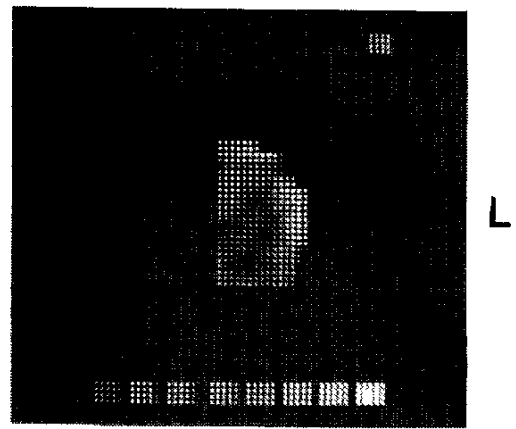

L-STA comp,

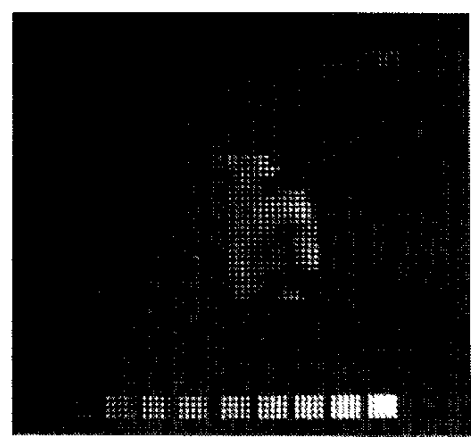

decreased area

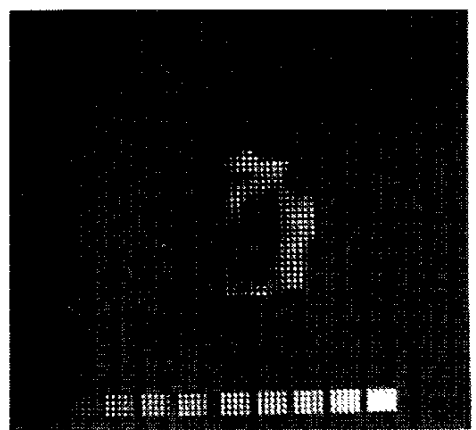

Fig. 2 Preoperative functional image of regional cerebral blood flow in Case 1 showing a decreased area of blood flow when the left superficial temporal artery (STA) was digitally compressed.

anterior branch of the STA was carefully preserved during the operation. The postoperative course was uneventful. Serial cerebral angiograms on the 7 th postoperative day demonstrated fairly good filling of blood vessels in the left MCA region through the posterior branch of the left STA (Fig. 3-a). The extrato intracranial channels from the anterior branch of the left STA to the left ophthalmic artery were functioning. Fig. 4 illustrates the
Doppler blood flow sonagrams obtained from the posterior branch of the STA, showing a shift from an external to an internal carotid pattern after the anastomosis. This shift suggested that the blood flow through the posterior branch of STA began to perfuse the internal carotid artery system.

The patient has been asymptomatic since surgery and mild diabetes has been well controlled with medical treatment. 


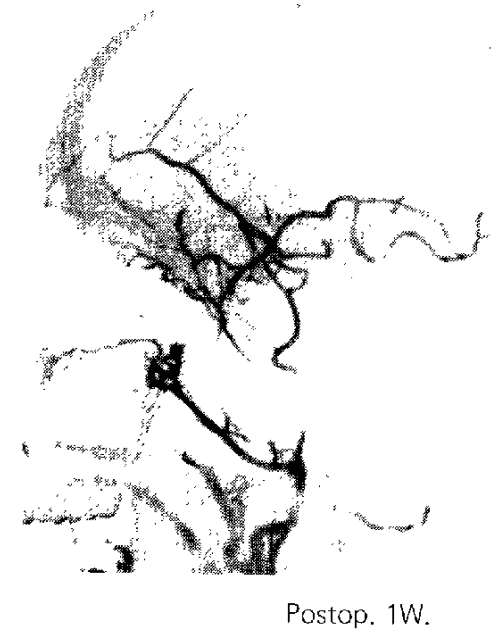

(a)

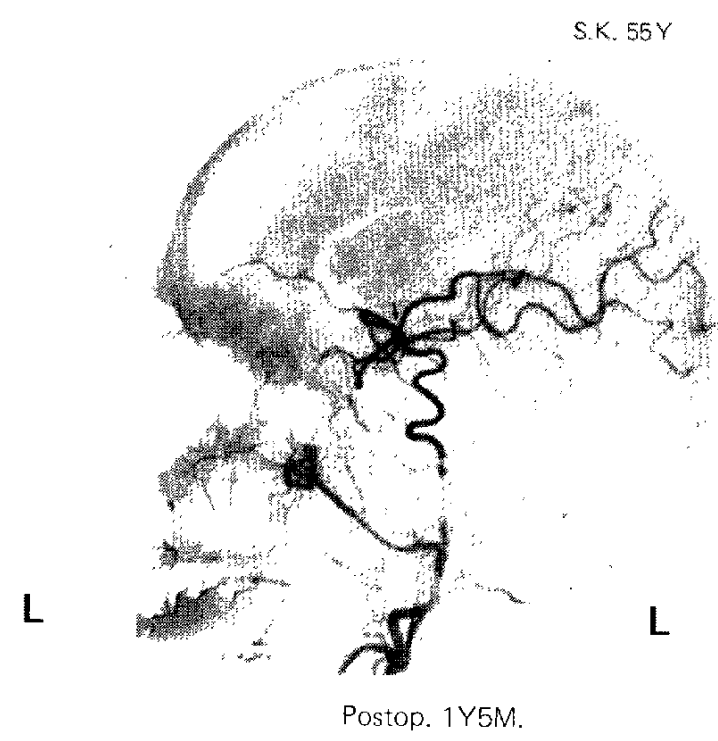

(b)

Fig. 3 a) Lateral view of the left postoperative angiogram showing patent anastomosis with good filling in the temporal territory of the left middle cerebral artery. The anterior branch of the STA was still functioning as an extra- to intracranial collateral channel.

b) Lateral view of follow-up angiogram. The filling of the temporal branches of the middle cerebral artery through the surgical bypass increased. The collateral channel through the anterior branch of the STA became atrophic. Arrows indicate the site of microanastomosis using the posterior branch of the STA on the left side.
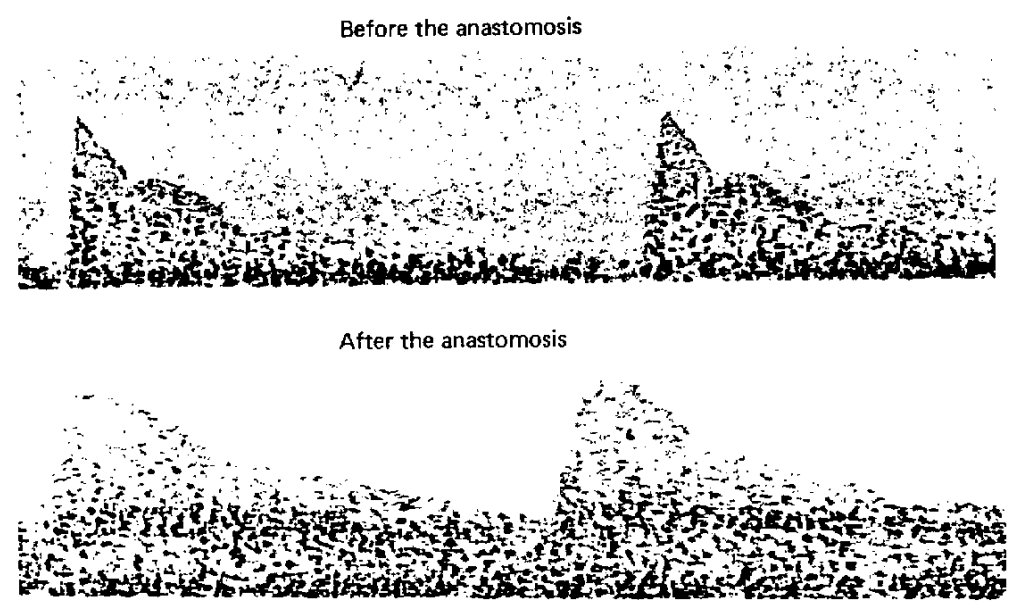

(S.K. 54Y. male)

Fig. 4 Changes in the flow pattern of an ultrasonic sonagram obtained from the posterior branch of the left STA.

Upper: Preoperative rheogram of the posterjor branch of the STA showing the external carotid patterns.

Lower: Postoperative rheogram of the same branch showing the internal carotid patterns. 
Subsequent postoperative angiography was performed one and a half years after the surgery (Fig. 3-b). The filling of blood vessels in the left $\mathrm{MCA}$ region through the posterior branch of the left STA had increased. The STA had dilated in comparison with the early postoperative period. On the other hand, the anterior branch of the STA had become narrow and atrophic. The collateral circulation through the left ophthalmic artery had apparently decreased; the reverse blood flow of the left ophthalmic artery was no longer detected by directional Doppler flowmetry. In contrast, the flow velocity of the posterior branch of the
STA was markedly increased. These findings of Doppler flowmetry corresponded well with those of the angiography.

\section{Case 2}

A 38 year-old left-handed male had a history of weakness of his left upper extremity for several months in 1975. Cerebral angiograms revealed occlusions of bilateral cervical internal carotid arteries at their bifurcations. His weakness improved and no surgical treatment was performed at that time. By mid-April 1976, the weakness of the left upper extremity recurred and extended progressively to the left
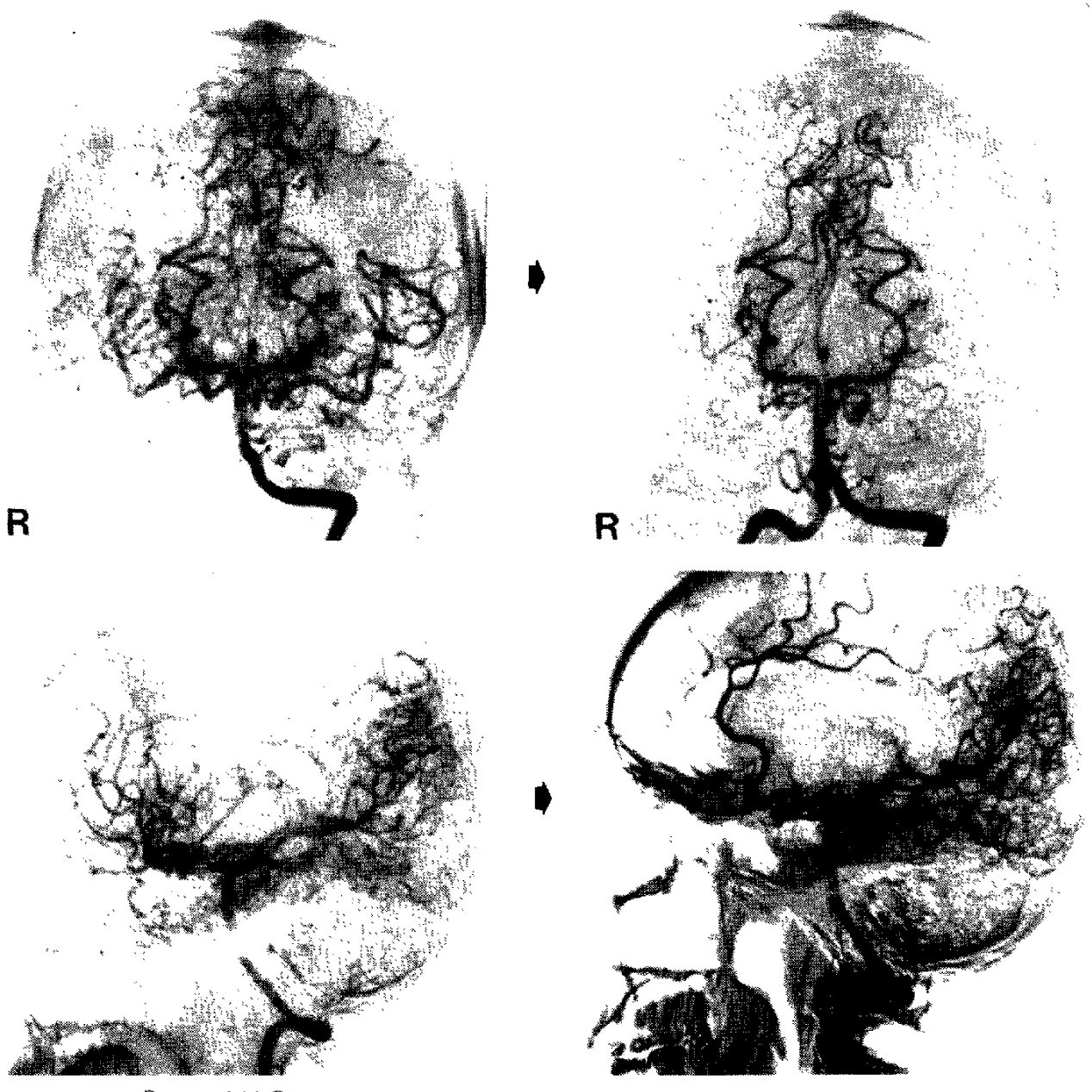

Preop. VAG

$5-20-76$

Postop. 8M.

$1-19-77$

Fig. 5 Changes of cerebral circulation after STA-MCA anastomosis in a case of bilateral occlusion of the internal carotid arteries (Case 2).

a) In contrast to preoperative vertebral angiograms, bilateral MCA territories were not visualized postoperatively. These territories were flled through the surgical bypass via STAs. 
lower extremity. He was admitted to Osaka University Hospital on May 13, 1976. Neurological examinations indicated left hemiparesis with slight impairment of the left lower cranial nerves. Laboratory examinations were negative except for mild hypercholesteremia. A small low density area with ring enhancement was demonstrated in the right frontoparietal area in CT scanning. In an EEG examination, focal slow activity was noted in the right frontal region. Preoperative cerebral angiograms confirmed the previous findings. The internal carotid arteries were completely occluded bilaterally at the cervical level. The anterior and middle cerebral arteries on both sides were filled through the vertebral arteries (Fig. 5-a). The posterior pericallosal arteries were visualized as collateral routes from posterior to anterior circulation.

STA-MCA anastomosis was carried out on the right side and then on the left side after an interval of 3 weeks. A small focal ischemic change was observed on the right cortical surface. The progressive course of neurological disorders was arrested after the surgery and hemiparesis improved to some extent by rehabilitation.

Postoperative angiography was performed on the 7th day after each operation. Anastomoses were proved to be patent (Figs. 5-b and
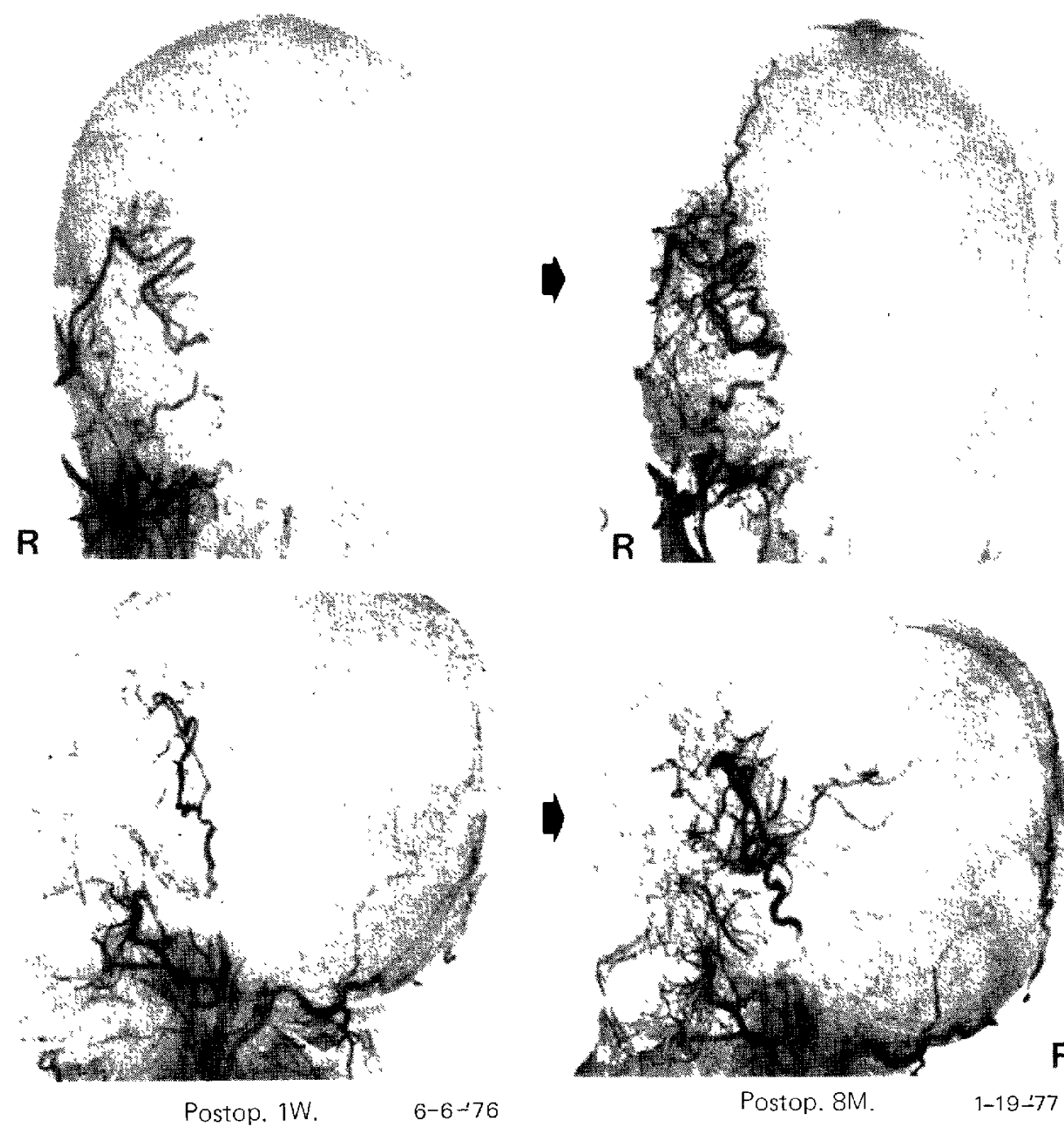

Fig. 5 b) Right postoperative external carotid angiograms. The STA had dilated and blood flow had increased. 

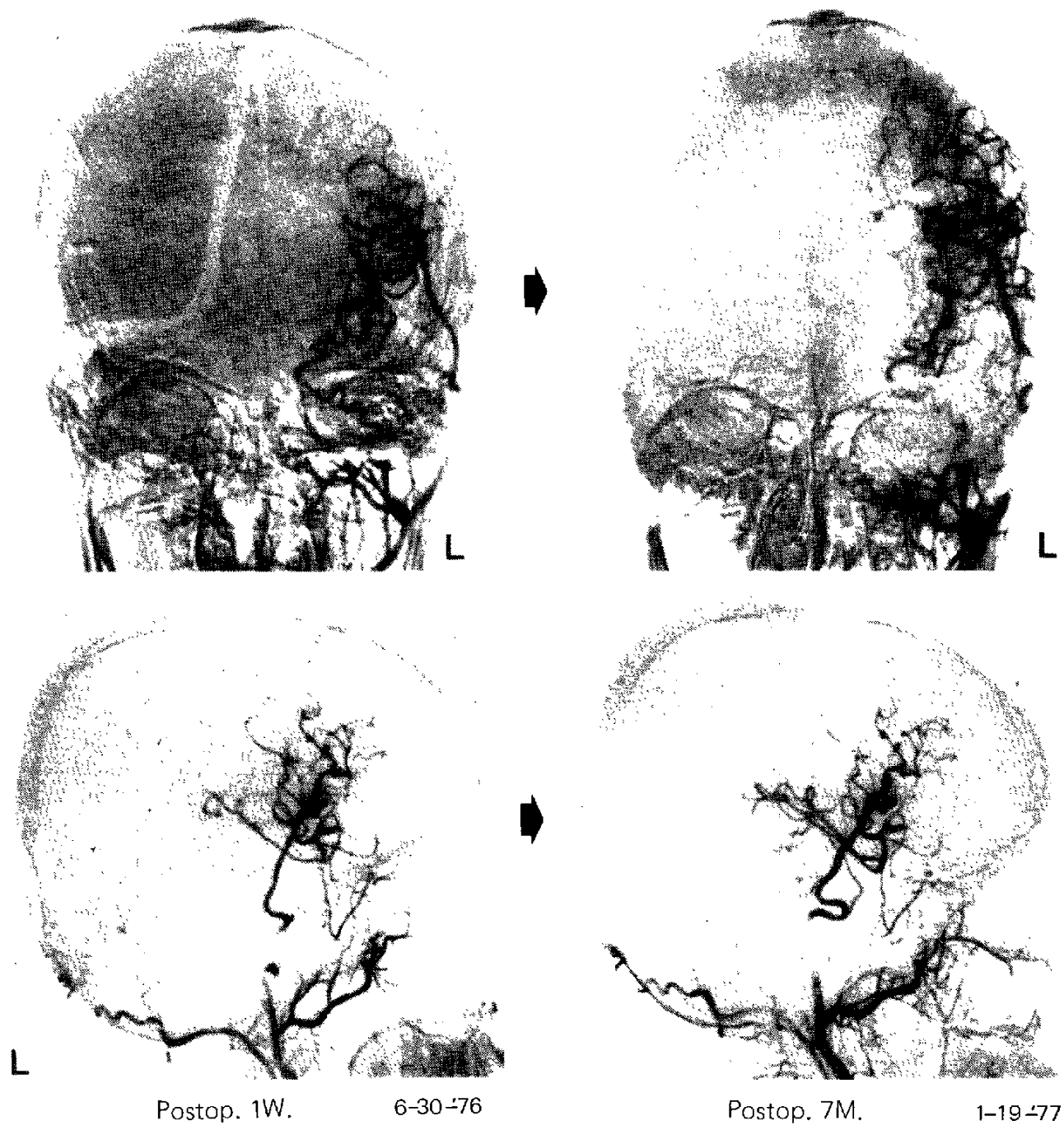

Fig. 5 c) Left postoperative external carotid angiograms. Collateral flow had increased through the surgical bypass.

5-c). Follow-up angiograms obtained 8 months after right side surgery and 7 months after left side surgery demonstrated dilated donor arteries and anastomoses on both sides. MCA territories drained through the STAs were markedly extended. In postoperative vertebral angiograms, the anterior cerebral arteries as well as posterior circulation were visualized, but arteries in the MCA territories on both sides were not. These findings indicated that blood to the MCA territories on both sides was drained only through the microsurgically constructed new collateral channels and that the changes of intravascular pressure balance resulted in redistribution of cerebral circulation
(Fig. 5-a.)

\section{Discussion}

It is well known that when cerebral blood flow through primary pathways is reduced by occlusion of aortocranial vessels, focal ischemia takes place in the brain and compensatory collateral circulation is developed to relieve it. The degree and extent of the development of collateral circulation play a role in the development of neurological disorders and influence the clinical course in strokes.

The collateral channels which drain into the brain are divided mainly into two routes ${ }^{2,4)}$; 
(1) intracranial/intracerebral anastomoses: basal anastomoses forming the circle of Willis, pericerebral leptomeningeal anastomoses ${ }^{14)}$ and intracerebral peripheral anastomoses such as the thalamoperforating, lenticulostriate, posterior pericallosal arteries; and (2) extra- to intracranial routes: collaterals through muscular branches of the vertebral artery, occipital artery, meningeal arteries and ophthalmic artery with retrograde flow through the external carotid group. Three routes have been reported ${ }^{4,10}$ as main anastomotic pathways via the ophthalmic artery. As shown in Fig. 6, the anterior branch of the STA is one of the three pathways and, in case of occlusion of the internal carotid artery, this route plays a very important role as a collateral channel for intracerebral circulation.

The purpose of STA-MCA anastomosis is to provide additional extra- to intracranial collateral pathways surgically. ${ }^{8}$ Our criteria for surgical indication are: (1) repeated ischemic attacks with complete or total recovery, a progressing stroke, or a minor completed stroke, (2) an angiographically detected occlusive lesion that is inaccessible or not suitable for a direct operation, (3) absence of massive infarction in CT scanning and (4) global or focal decreased $\mathrm{rCBF}$.

In the first case, evidence obtained from preoperative cerebral angiograms, rCBF measurement by the STA compression test and Doppler flowmetry of the ophthalmic artery suggested that the blood flow of the ophthalmic artery via the anterior branch of the left STA played an important role as a collateral channel into the ischemic brain. This collateral route was, therefore, carefully preserved during the surgery and only the posterior branch of the STA was used as a donor artery for STA-MCA anastomosis.

In the early postoperative period, marked spasms and/or narrowing of vessels around the anastomosis have been observed frequently. "On the early postoperative angiograms, eight out of 22 operations in our series showed spastic and malfunctioning appearance of the vessels around the microanastomoses (Fig. 7). These findings suggest that the surgically reconstructed bypass may not be functioning well in the early postoperative period. Surgical disconnection of preexisting

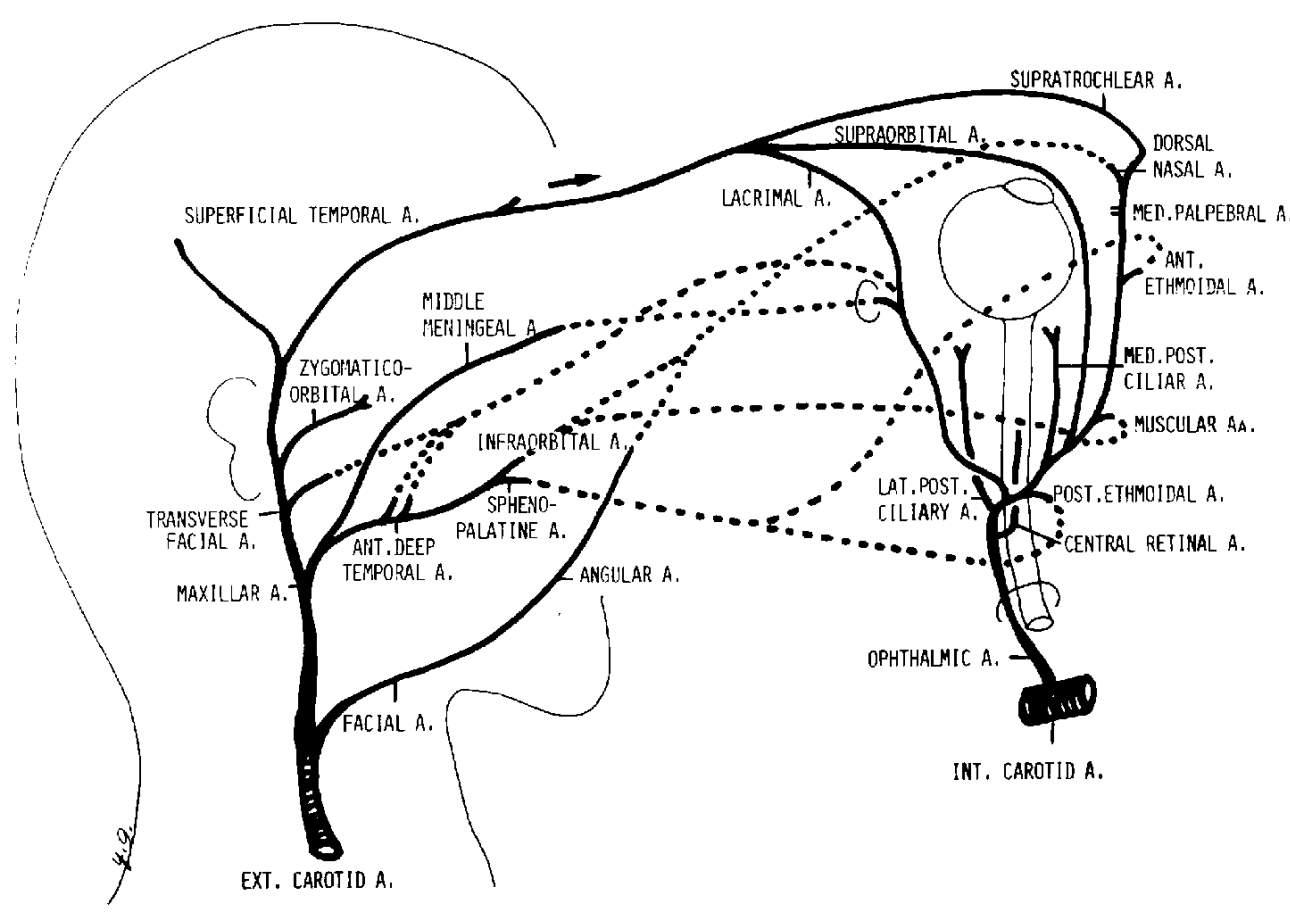

Fig. 6 Collateral pathways from the external carotid to the intracranial internal carotid artery via the ophthalmic artery. 

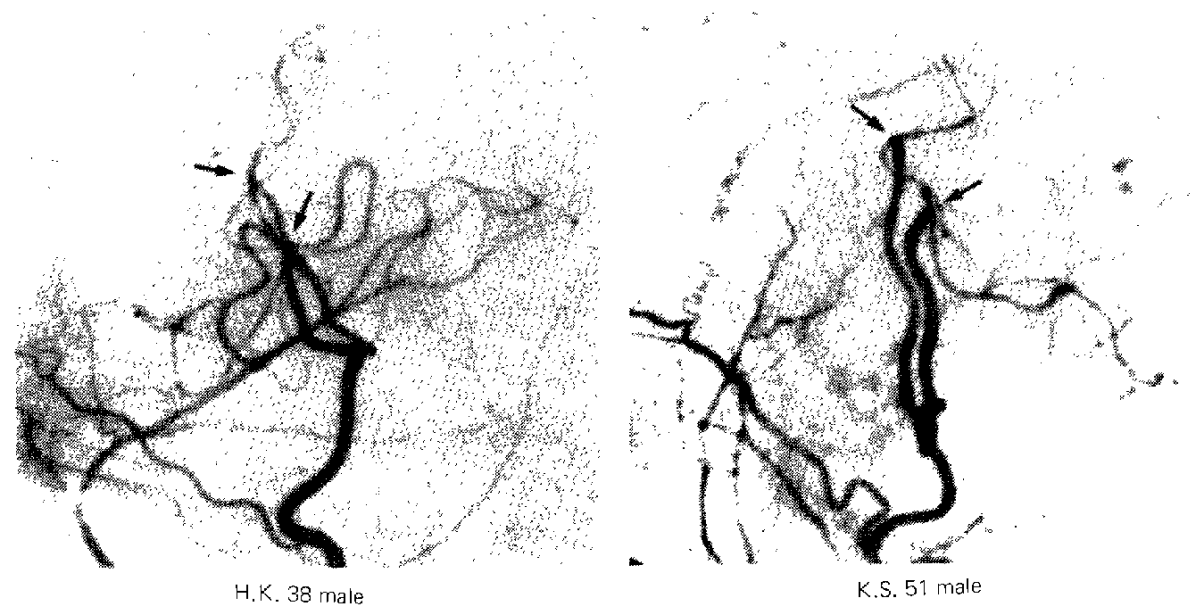

Fig. 7 Spasms around the microsurgical anastomoses in the early postoperative period. Lateral view of angiograms on the 10th postoperative day in either case demonstrated the patency of the bypasses and vasospasms around the sites of anastomoses.

significant collaterals, even if for the purpose of bypass formation, may cause aggravation of ischemia in the brain and further deterioration of neurological symptoms. We would like to stress, therefore, that preexisting collateral circulatory routes should be preserved during STA-MCA anastomosis for ischemic cerebral vascular diseases.

In 10 out of 20 cases in our series, postoperative electroencephalography demonstrated the appearance of focal slow waves in the anterior temporal region on the side of operation. Such slow activities were temporary and disappeared within 2 to 3 months after the operation. This evidence suggests that local cortical activity is reversibly depressed during the early postoperative period, possibly due to a surgical procedure such as craniotomy per se.

Postoperative rCBF was decreased to some degree in three out of 16 cases in our series, although the neurological conditions improved after surgery in all cases. The measurement of rCBF in these three cases was performed within 3 weeks after surgery (Table 1). Schmiedek et al. ${ }^{12)}$ stated that in their rCBF studies a considerable period was required before the collateral effect of the bypass could be established, and therefore final assessment of the operative results could not be made for at least 3 months postoperatively. These observations support our view that the surgical anastomosis may not provide sufficient collateral channels in the early postoperative period. The time of examination after surgery is sure to influence the interpretation of the surgical results. ${ }^{6}$

The enlargement of donor artery following STA-MCA bypass surgery has been reported previously. ") Our long term follow-up observations clearly demonstrated that the donor STA dilated and blood flow increased with time, which indicated that this route developed to

Table 1 Postoperative changes in rCBF measurement and follow-up periods.

\begin{tabular}{ccccc}
\hline $\begin{array}{c}\text { Postop. Period } \\
\text { (months) }\end{array}$ & Increased & Unchanged & Decreased* & Total \\
\hline-1 & 1 & 2 & 3 & 6 \\
$1-2$ & 2 & 3 & 0 & 5 \\
$2-3$ & 1 & 1 & 0 & 2 \\
$3-$ & 0 & 3 & 0 & 3 \\
\hline Total & 4 & 9 & 3 & 16 cases \\
\hline
\end{tabular}

* In all three cases rCBF was measured within 3 weeks after the operation. 
become a main collateral circulatory pathway taking the place of occluded vessels. On the other hand, it was obvious that some preexisting collateral channels, such as the routes of the ophthalmic artery in Case 1, tended to diminish after surgery.

Progressive changes in collateral channels may be caused by the pressure difference between the recipient artery and the donor artery, the tissue adaptation of the channels and the metabolic demand of ischemic brain tissue, ${ }^{11)}$ etc. Surgical bypass may therefore be of limited value as a collateral channel in the early postoperative period, but it does play an important and sufficient role as a main collateral route subsequently and may come to improve the patient's condition and prevent cerebral ischemic episodes in the future.

\section{Conclusions}

From the experience with 20 cases of STAMCA anastomosis for the treatment of cerebral ischemia, the following conclusions were drawn.

1. Redistribution of cerebral circulation takes place following surgery, and changes progressively.

2. The surgically constructed collateral pathway dilates with time in most of the cases. The function of the bypass, however, might be of limited value in the early postoperative period in certain cases.

3. Although some of the preexisting extrato intracranial collateral channels become atrophic following surgery, they must be allowed to remain in the early postoperative period when surgical bypass is not yet functioning sufficiently.

4. From the point of view of collateral circulation, the anterior branch of the STA should be preserved if it is functioning as one of the main extra- to intracranial collateral pathways preoperatively.

\section{Acknowledgment}

We wish to express our thanks to the staff of the Department of Neurosurgery, the cerebral circuratory group of $1 \mathrm{st}$ Department of Internal Medicine and the Department of Neuroradiology, Osaka University Hospital.
This paper was presented in part at the 35 th and 37th Annual Meetings of the Japan Neurosurgical Society in 1976 and 1978, and the 8th Japan Congress of the Surgical Treatment of Strokes in 1979.

\section{References}

1) Anderson, R. E., Reichman, O. H. and Davis, D. O.: Radiological evaluation of temporal artery-middle cerebral artery anastomosis. Radiology 113: 73-79, 1974.

2) Azar-Kia, B., Palacio, E.: Collateral circulation of the brain. pp 132-144, In Fein and Reichman (eds): Microvascular Anastomosis for Cerebral Ischemia. Springer-Verlag, New York, 1974.

3) Donaghy, R. P. M.: Patch and by-pass in microangeinal surgery. pp 75-86, In Donaghy and Yasargil (eds): Microvascular Surgery. C. V. Mosby, St. Louis, 1967.

4) Hayreh, S. S.: The ophthalmic artery. I. Normal gross anatomy. pp 1333-1350, In Newton and Potts (eds): Radiology of the Skull and Brain. C. V. Mosby, St. Louis, 1974.

5) Iwata, Y. and Hayakawa, T.: Surgical technique of STA-MCA anastomosis for the treatment of cerebral ischemia. Operation 31 : 1235-1240, 1977 (in Japanese).

6) Iwata, Y., Mukawa, J., Mogami, H., Hayakawa, T., Nukada, T., Imaizumi, $M$. and Kimura, K.: The study of preoperative selection of examinations and their postoperative evaluation period in STA-MCA anastomosis for the treatment of cerebral ischemia. Proceedings of 8th Japan Surgical Treatment of Stroke. Neuron-sha, Kawasaki, 1979, pp 113-118.

7) Khodadad, G.: Transient postoperative occlusion of the superficial temporal-middle cerebral artery branch anastomosis; spasm, swelling, or thrombosis. Surg Neurol 3: 341-345, 1975.

8) Lee, M. C., Ausman, J. I., Geiger, J. D., et al.: Superficial temporal to middle cerebral artery anastomosis. Arch Neurol 36: 1-4, 1979.

9) McDowell, F. H.: The extracranial/intracranial bypass study. Stroke 8: 545, 1977.

10) Mishkin, M. M. and Schreiber, M. N.: Collateral circulation. In Newton and Potts (eds): Radiology of the Skull and Brain. C. V. Mosby, St. Louis, 1974, 2(4): 2344-2374.

11) Reichman, O. H.: Cited by Millikan, C. H., McDowell, F. H.: Summary of the 10th Princeton conference on cerebral vascular diseases. Stroke 7: 238, 1976. 
12) Schmiedek, P., Gratzl, O., Steinhof, H., et al.: Blood flow and cerebral revascularization. Clin Neurosurg 23: 270-286, 1976.

13) Takano, T., Kimura, K., Sugitani, Y., et al.: Functional image of regional cerebral blood flow (Method and clinical applications). Acta Neurol Scand [Suppl] 64, 56: 440-441, 1977.

14) Van den Bergh, R. and vander Eecken, H.: Anatomy and embryology of cerebral circulation. Prog Brain Res 30: 1-25, 1968.
15) Yasargil, M. G.: Experimental small vessel surgery in the dog including patching and grafting of cerebral vessels and the formation of functional extra-intracranial shunts. pp 87-126, In Donaghy and Yasargil (eds): Microvascular Surgery. C. V. Mosby, St. Louis, 1970.

16) Yasargil, M. G., Krayenbühl, H. and Jacobson, J. H.: Microneurosurgical arterial reconstruction. Surgery 67: 221-233, 1970. 